\title{
Dislocation nucleation governed softening and maximum strength in nano-twinned metals
}

\author{
Xiaoyan $\mathrm{Li}^{1}$, Yujie $\mathrm{Wei}^{2} \dagger$, Lei $\mathrm{Lu}^{3}, \mathrm{Ke} \mathrm{Lu}^{3}$ \& Huajian Gao ${ }^{1}$
}

In conventional metals, there is plenty of space for dislocations-line defects whose motion results in permanent material deformationto multiply, so that the metal strengths are controlled by dislocation interactions with grain boundaries ${ }^{1,2}$ and other obstacles ${ }^{3,4}$. For nanostructured materials, in contrast, dislocation multiplication is severely confined by the nanometre-scale geometries so that continued plasticity can be expected to be source-controlled. Nanograined polycrystalline materials were found to be strong but brittle $^{5-9}$, because both nucleation and motion of dislocations are effectively suppressed by the nanoscale crystallites. Here we report a dislocation-nucleation-controlled mechanism in nano-twinned metals ${ }^{10,11}$ in which there are plenty of dislocation nucleation sites but dislocation motion is not confined. We show that dislocation nucleation governs the strength of such materials, resulting in their softening below a critical twin thickness. Large-scale molecular dynamics simulations and a kinetic theory of dislocation nucleation in nano-twinned metals show that there exists a transition in deformation mechanism, occurring at a critical twin-boundary spacing for which strength is maximized. At this point, the classical Hall-Petch type of strengthening due to dislocation pile-up and cutting through twin planes switches to a dislocation-nucleationcontrolled softening mechanism with twin-boundary migration resulting from nucleation and motion of partial dislocations parallel to the twin planes. Most previous studies ${ }^{12,13}$ did not consider a sufficient range of twin thickness and therefore missed this strength-softening regime. The simulations indicate that the critical twin-boundary spacing for the onset of softening in nano-twinned copper and the maximum strength depend on the grain size: the smaller the grain size, the smaller the critical twinboundary spacing, and the higher the maximum strength of the material.

Ultrafine-grained $\mathrm{Cu}$ with nanoscale thin twins embedded in individual grains has recently been synthesized, achieving a strength increase by a factor of 7 to 10 relative to conventional coarse-grained polycrystalline $\mathrm{Cu}$, as well as considerable ductility and high electrical conductivity $^{10,11}$. More interestingly, the strength of such nanotwinned $\mathrm{Cu}$ first increases as the twin-boundary spacing $\lambda$ decreases, reaching a maximal strength at $\lambda=15 \mathrm{~nm}$, then decreases as $\lambda$ is further reduced ${ }^{11}$. The trend of increasing strength in nano-twinned ultrafine-grained $\mathrm{Cu}$ with decreasing $\lambda$ can be relatively well explained by the Hall-Petch effect because the twin planes can serve as barriers to dislocations gliding on inclined slip planes. However, the strength softening with a further decrease of $\lambda$ from $15 \mathrm{~nm}$ to $4 \mathrm{~nm}$ is intriguing.

For nanocrystalline metals without nano-twin substructures, molecular dynamics simulations ${ }^{6-9}$ have shown a strength softening mechanism as grain size is reduced to about $10 \mathrm{~nm}$ in $\mathrm{Cu}$, which has been attributed to a transition from dislocation-mediated plastic deformation to grain-boundary-associated mechanisms such as grain-boundary sliding, grain-boundary diffusion and grain rotation $^{5,14-18}$. In nano-twinned ultrafine-grained $\mathrm{Cu}$, the observed strength softening cannot be attributed to grain-boundary-associated mechanisms for the following reasons: (1) twin planes are coherent in nature, and shearing along them is as difficult as most other atomic planes; and (2) grain sizes and grain-boundary properties for samples with different twin thicknesses are similar ${ }^{10,11}$. Although it has been speculated that the softening in nano-twinned ultrafine-grained $\mathrm{Cu}$ might be caused by pre-existing dislocations potentially acting as easy dislocation sources in samples with smaller $\lambda$ (ref. 11), it remains difficult to reveal the detailed interactions between dislocations and twin planes using post-mortem microstructure observations. Here we use massively parallel atomistic simulations to investigate the effect of twin thickness on the deformation mechanisms in nano-twinned $\mathrm{Cu}$. The simulations show that the strength softening in nano-twinned $\mathrm{Cu}$ is governed by dislocation nucleation at grain boundary-twin intersections. Such dislocation-nucleation-controlled strength is rarely observed because dislocations can easily multiply given sufficient space. A possible exception is the strengthening mechanism of microand nano-pillars, which has been attributed to the increasing difficulty of dislocation nucleation and multiplication as the structure dimension is reduced ${ }^{19-22}$.

Deformation of nano-twinned crystals has previously been investigated using molecular dynamics simulations ${ }^{23,24}$. To represent real material structures seen in experiments as closely as possible ${ }^{10,11}$, we have performed simulations on fully three-dimensional polycrystals with sub-grain nano-twins. Simulations reported in this study involve a total of 140 million atoms. Details about the simulations are given in the Methods. Stress versus strain curves for two different grain sizes $d=10 \mathrm{~nm}$ and $20 \mathrm{~nm}$, and for various values of the twinboundary spacing are shown in Fig. 1a. It can be seen in Fig. 1b that the average flow stress first increases with decreasing twin-boundary spacing $\lambda$, reaching a maximum at a critical twin-boundary spacing, and then drops progressively with further decreasing $\lambda$, in qualitative agreement with experimental observations ${ }^{11}$.

Deformation patterns of two samples with different twin-boundary spacing but the same grain size $d=20 \mathrm{~nm}$ are shown in Fig. 2 (see also Supplementary Discussion 1). The result for the sample with $\lambda=1.25 \mathrm{~nm}$ at $10 \%$ strain is shown in Fig. 2a where plastic deformation is dominated by twin-boundary migration: partial dislocations are observed to nucleate at grain boundaries and glide along the twin planes (Supplementary Discussion 1, see also Supplementary Fig. 6), resulting in a change of twin-boundary spacing. Dislocations intersecting with twin planes are rarely seen here. The observed twinboundary migration induced by the motion of leading partial dislocations is consistent with previous investigations $s^{3,25-27}$. In the sample with $\lambda=6.25 \mathrm{~nm}$ (Fig. 2b), plenty of dislocations intersecting twin 


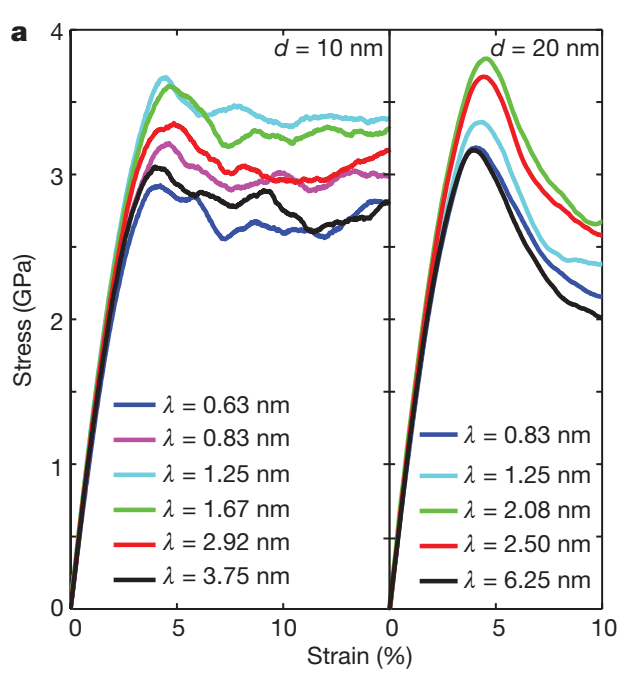

b

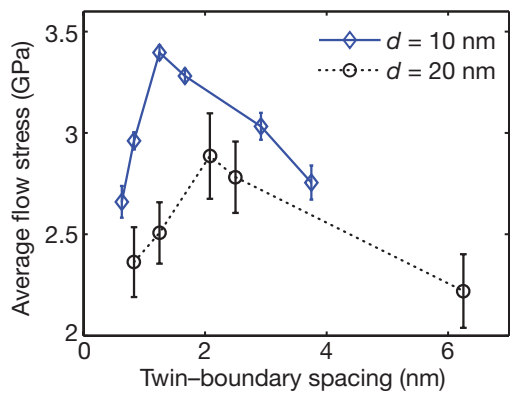

Figure 1 | Stress-strain relations and flow stress from molecular dynamics simulations of nano-twinned $\mathrm{Cu}$. a, Simulated stress-strain curves for nanotwinned $\mathrm{Cu}$ with different values of twin-boundary spacing for two grain sizes $d=10 \mathrm{~nm}$ (left) and $d=20 \mathrm{~nm}$ (right). $\mathrm{b}$, Averaged flow stress from an engineering strain of $6-15 \%$ for $d=10 \mathrm{~nm}$ and $6-10 \%$ for $d=20 \mathrm{~nm}$. The flow stress first rises as the twin-boundary spacing decreases, reaching a maximum at a critical twin-boundary spacing, and then drops as twins are further narrowed. Error bars represent the standard deviation from statistical analyses with $n=459(d=10 \mathrm{~nm})$ and $n=208(d=20 \mathrm{~nm})$ sampling points.

planes were observed. In the latter case, plastic deformation is dominated by partial dislocations emitted from grain boundaries cutting into the neighbouring twin planes, and twin-boundary migration plays a much smaller part in accommodating plastic deformation. A diagram for the nucleation of dislocations parallel or inclined to twin boundaries is shown in Supplementary Fig. 2.

Typical dislocation structures in the interior of a grain are shown in Fig. 3. Figure 3a shows several partial dislocations nucleated from grain boundaries and moving parallel to twin boundaries in the $\lambda=1.25 \mathrm{~nm}$ sample at $4 \%$ strain. As the sample was further loaded to $7 \%$ strain (Fig. 3b), most dislocation lines were parallel to, and rarely seen to intersect with, the twin planes. In contrast, for the sample with the same grain size but larger twin-boundary spacing $\lambda=6.25 \mathrm{~nm}$ at $5.4 \%$ strain, dislocations were commonly seen to nucleate from grain boundaries and intersect twin boundaries (Fig. 3c). In the samples with grain size $d=10 \mathrm{~nm}$, we found the same transition in deformation mechanism as the twin-boundary spacing is reduced. The evolution of the number of hexagonal close packed atoms associated with stacking faults shown in Supplementary Fig. 3 also confirms that there exists a transition in deformation mechanism at a critical twin-boundary spacing.

To confirm that the same strength softening phenomenon also exists in quasi-three-dimensional simulations, we also performed simulations with columnar grain structures (see Supplementary Discussion 2). We considered samples with average grain size $d=70 \mathrm{~nm}$ and twin-boundary spacing $\lambda=2 \mathrm{~nm}, 5 \mathrm{~nm}, 10 \mathrm{~nm}, 15 \mathrm{~nm}, 20 \mathrm{~nm}$ and $25 \mathrm{~nm}$. The same transition from motion of discrete dislocations

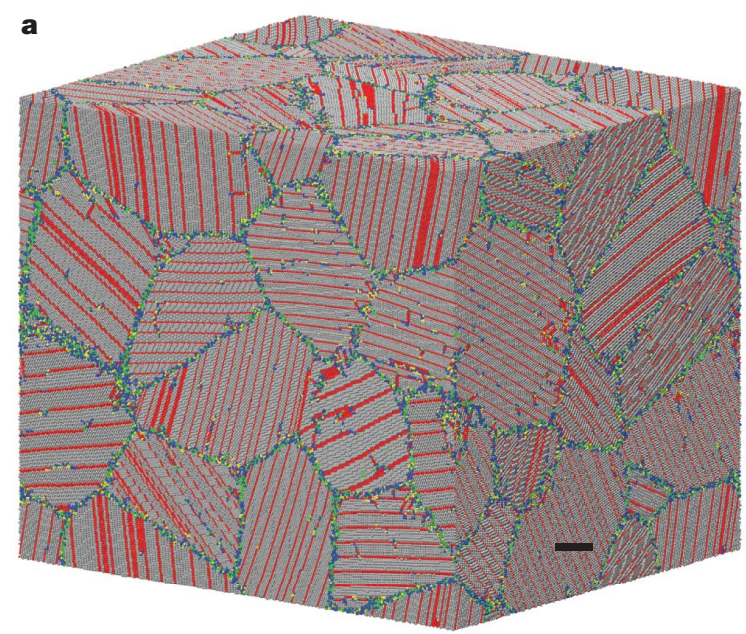

b

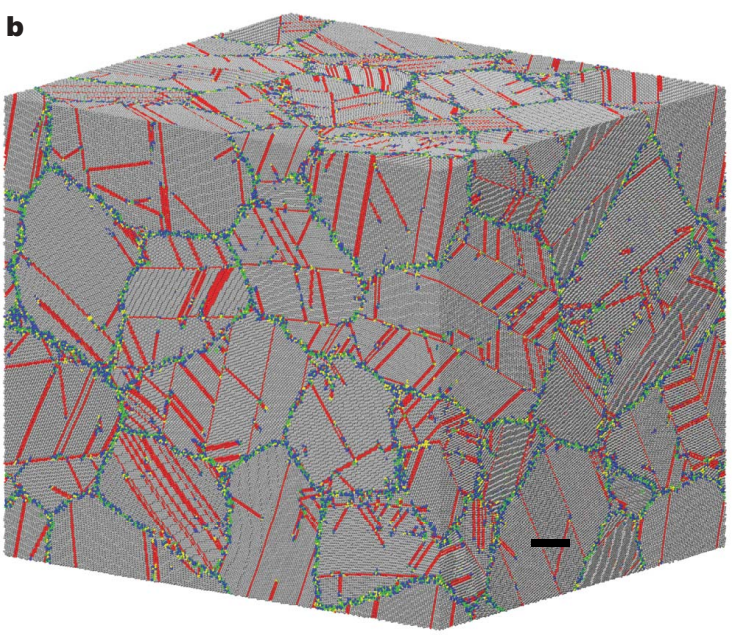

Figure 2 | Simulated deformation patterns in nano-twinned samples with grain size $\boldsymbol{d}=\mathbf{2 0} \mathrm{nm}$ at $\mathbf{1 0 \%}$ strain. (Scale bars, $5 \mathrm{~nm}$.) Samples with twinboundary spacing $\lambda=1.25 \mathrm{~nm}$ (a) and $\lambda=6.25 \mathrm{~nm}(\mathrm{~b})$. In a, plastic deformation is dominated by partial dislocations gliding parallel to twin planes, whereas in $\mathbf{b}$, dislocations cutting across twin planes is the controlling deformation mechanism.

intersecting with twin planes to that of partial dislocations parallel to the twin planes was observed as the twin-boundary spacing was reduced (see Supplementary Fig. 8).

Interestingly, it is noted that the transition happens at different values of twin-boundary spacing in samples with different grain sizes, and the corresponding maximal strengths of the material are also distinct. We will next investigate theoretically why dislocation nucleation at grain boundary-twin intersections would lead to the softening of material below a critical twin-boundary spacing.

The strength of crystalline metals is controlled by both initial defects (mainly dislocations) and the capability of the material to nucleate new defects to carry plastic deformation. In nano-twinned $\mathrm{Cu}$, plastic strains by pre-existing dislocations may be estimated using $\varepsilon=\rho_{0} b d /$ $M$ (ref. 11). Taking the initial dislocation density to be $\rho_{0} \approx 10^{14} \mathrm{~m}^{-2}$ in samples with $\lambda=4 \mathrm{~nm}$, the magnitude of the Burgers vector for Shockley partial dislocations in $\mathrm{Cu}$ to be $b=0.147 \mathrm{~nm}$, grain size to be $d=500 \mathrm{~nm}$, and $M=6$ to be twice the Taylor factor owing to the directionality of partial dislocations, we obtain roughly a $0.1 \%$ of plastic strain by initial dislocations if those dislocations seen under transmission electron microscopy are mobile. The pre-existing dislocations could consequently influence the $0.2 \%$ yield strength and hence significantly affect the experimentally observed strength softening as the twin-boundary spacing decreases. Molecular dynamics simulations of nano-indentation testing ${ }^{28}$ also support the conjecture that pre-existing defects in twin structures could cause strength softening. 

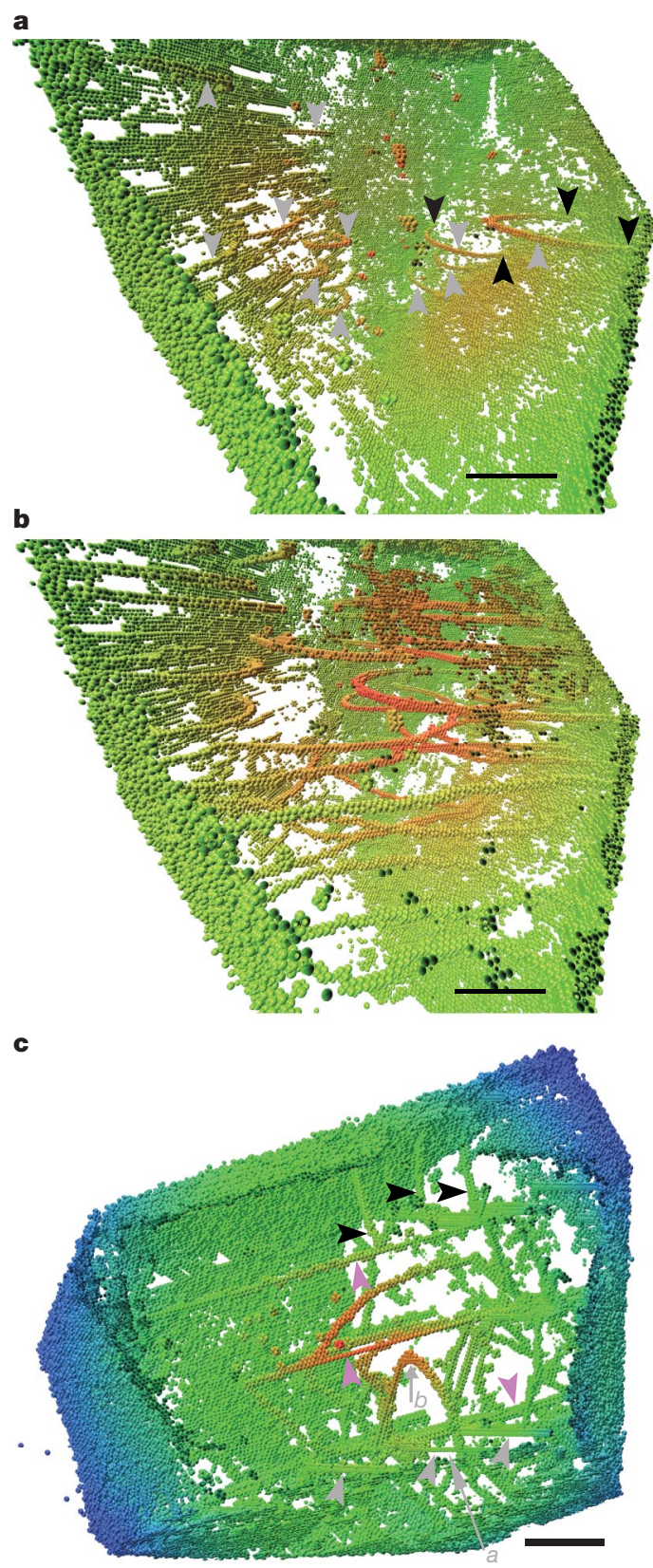

Figure 3 | Dislocation structures in $\boldsymbol{d}=\mathbf{2 0} \mathbf{n m}$ sample. (Scale bars, $5 \mathrm{~nm}$.) Colouring is based on distance of atoms to grain centre. $\mathbf{a}$, In the $\lambda=1.25 \mathrm{~nm}$ sample at $4 \%$ strain, dislocations (grey and black arrows) nucleate from grain boundaries and move parallel to twin boundaries. $\mathbf{b}$, In the $\lambda=1.25 \mathrm{~nm}$ sample at $7 \%$ strain, dislocations remain parallel to twin boundaries. c, In the $\lambda=6.25 \mathrm{~nm}$ sample at $5.4 \%$ strain, dislocations intersect (black and grey arrows) or lie parallel to (purple arrows) twin boundaries. One dislocation cuts through a twin boundary, leaving behind a residual partial label ' $a$ ' on the twin boundary and a transmission dislocation ' $\mathrm{b}$ '.

In our large-scale simulations, however, the samples are initially dislocation-free and subsequently exhibit a rapid increase in dislocation density to $\sim 10^{16} \mathrm{~m}^{-2}$ during plastic deformation (see Supplementary Fig. 4). This rapid increase of dislocation density is comparable to the experimental observation that dislocation density increased from $\sim 10^{14} \mathrm{~m}^{-2}$ to $\sim 10^{16} \mathrm{~m}^{-2}$ as strain was increased to about $20 \%$ in the nano-twinned Cu sample with $\lambda=4 \mathrm{~nm}$ (ref. 11).

Our simulations have provided a number of insights into the mechanism of strength softening in nano-twinned $\mathrm{Cu}$ : (1) an initially dislocation-free nano-twinned metal exhibits strength softening below a critical twin thickness; (2) in the strength softening regime, dislocation nucleation occurs primarily at the grain boundary-twin intersections; (3) there exists significant stress concentration at the grain boundary-twin intersection (Supplementary Discussion 3, see also Supplementary Fig. 9). These results, combined with experimental observations, imply that dislocation nucleation should govern the observed strength softening below a critical twin-boundary spacing. On the basis of these critical insights, we have formulated a theory of strength softening in nano-twinned metals by considering the kinetics of dislocation nucleation and available source density in such materials (for details see Supplementary Discussion 4). In this theory, the strength of the material depends on both twin-boundary spacing $\lambda$ and grain size $d$ as:

$$
\tau=\frac{\Delta U}{S V^{*}}-\frac{k_{\mathrm{B}} T}{S V^{*}} \ln \left(\frac{d}{\lambda} \frac{v_{\mathrm{D}}}{\dot{\varepsilon}}\right)
$$

where $\Delta U$ is the activation energy, $S$ is a factor representing local stress concentration and geometry, $V^{*}$ is the activation volume, $k_{\mathrm{B}}$ and $T$ are the Boltzmann constant and temperature, $v_{\mathrm{D}}$ is the Debye frequency, and $\dot{\varepsilon}$ is the macroscopic strain rate. Figure 4 shows a comparison of the yield stress of nano-twinned ultrafine-grained $\mathrm{Cu}$ from experimental data, the model predictions based on equation (1) and our molecular dynamics simulation results. The softening behaviour seen in the simulations below a critical twin-boundary spacing is well captured by the model. For a given grain size $d$, the junction of the $\tau$ versus $\lambda$ curve from equation (1) and the Hall-Petch relation indicates where the onset of strength softening occurs in a nano-twinned metal, provided that the Hall-Petch relation remains valid for twin-boundary spacing as small as several nanometres. As shown in Fig. 4, both simulations and the theory consistently show that dislocation nucleation governs the softening in nano-twinned metals, and that the onset of softening depends on the grain size: the smaller the grain size, the smaller the critical twin-boundary spacing, and the higher the maximum strength of the material. For samples with grain size $d=20 \mathrm{~nm}$ and $\lambda \approx 2-4 \mathrm{~nm}$, we predict a tensile yield strength around $2 \mathrm{GPa}$ in nano-twinned $\mathrm{Cu}$.

In conclusion, we have identified a dislocation-nucleationcontrolled softening mechanism in nano-twinned metals in which dislocation nucleation and storage are highly organized by the existing twins. Both the increased source density and increased twin boundaries for dislocation storage are essential to the observed

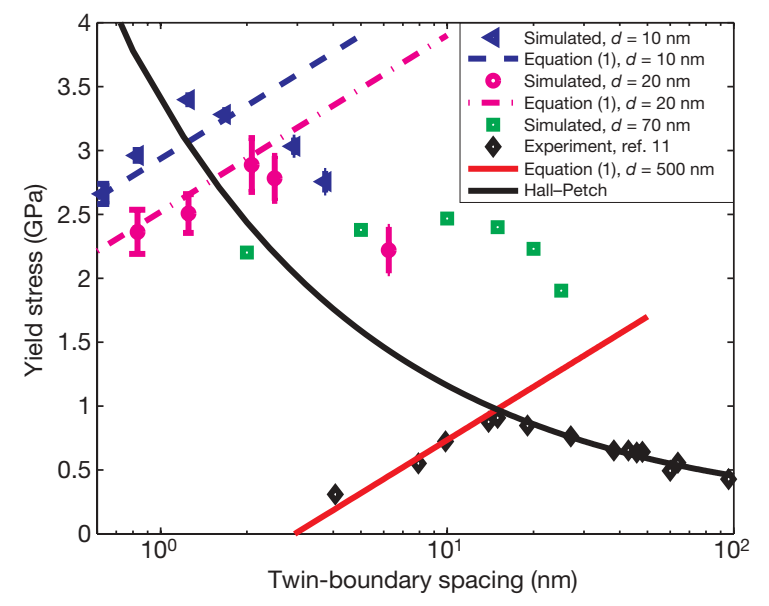

Figure 4 | Yield stress of nano-twinned $\mathrm{Cu}$ as a function of twin-boundary spacing at different grain sizes. Experimental data ${ }^{11}$ in the strengthincreasing region is fitted to the Hall-Petch equation $\sigma=\sigma_{0}+k / \sqrt{\lambda}$, where $\sigma_{0}=127.8 \mathrm{MPa}, k=3,266 \mathrm{MPa} \sqrt{\mathrm{nm}}$ and $\lambda$ is twin-boundary spacing in nanometres. Molecular dynamics simulation results (symbols) for yield stress (taken as the averaged flow stresses at strains larger than 6\%) for $d=10 \mathrm{~nm}, 20 \mathrm{~nm}$ and experimental data for $d=500 \mathrm{~nm}$, are shown together with the corresponding fitted curves from equation (1). The quasi-threedimensional simulation results for $d=70 \mathrm{~nm}$ are shown but not fitted because equation (1) is based on dislocation nucleation in threedimensional nano-twinned metals. Error bars are as in Fig. 1 b. 
strength softening in nano-twinned metals. Such softening mechanisms can happen in samples with or without pre-existing dislocations. Our simulations and theory show that the critical twin-boundary spacing for the dislocation-nucleation-controlled mechanism depends on grain sizes: the smaller the grain size, the smaller the critical twinboundary spacing, and the higher the maximum strength of the material. Equation (1), combined with the Hall-Petch relation, predicts the critical twin-boundary spacing for the onset of softening in nanotwinned metals. Given that both the Hall-Petch relation and equation (1) are not specific to particular metals, the conclusion should be generally applicable to nano-twinned face-centred cubic metals.

\section{METHODS SUMMARY}

The simulations are performed on three-dimensional polycrystal samples containing 27 randomly orientated Voronoi grains. In each grain, twins are inserted by mirroring a portion of the matrix with respect to a twin plane, as shown in the crystallographic diagram in Supplementary Fig. 1. The same Voronoi grain structure is scaled to obtain samples with different grain sizes and twin-boundary spacing. The crystallographic orientations of all grains are retained as twinboundary spacing and/or grain size change. The samples with $d=10 \mathrm{~nm}$ and $d=20 \mathrm{~nm}$ have dimensions of $30 \times 30 \times 30 \mathrm{~nm}^{3}$ and $60 \times 60 \times 60 \mathrm{~nm}^{3}$, containing about 2,300,000 and 18,500,000 atoms, respectively. For $d=10 \mathrm{~nm}$, six samples with initial uniform twins of spacing $\lambda=0.63 \mathrm{~nm}, 0.83 \mathrm{~nm}, 1.25 \mathrm{~nm}$, $1.67 \mathrm{~nm}, 2.92 \mathrm{~nm}$ and $3.75 \mathrm{~nm}$ are simulated. For $d=20 \mathrm{~nm}$, five samples with $\lambda=0.83 \mathrm{~nm}, 1.25 \mathrm{~nm}, 2.08 \mathrm{~nm}, 2.50 \mathrm{~nm}$ and $6.25 \mathrm{~nm}$ are simulated.

The embedded atom method potential ${ }^{29}$ for $\mathrm{Cu}$ was adopted. All simulations are performed at $300 \mathrm{~K}$ using a Nose-Hoover thermostat ${ }^{30}$. The multiple timestep algorithm ${ }^{31}$ was used with shorter and longer time steps of 2 femtoseconds and 6 femtoseconds, respectively. Periodic boundary conditions were imposed in all three directions. The sample is relaxed for 500 picoseconds before straining. During uniaxial tension, a $15 \%$ strain is applied to each sample over 750 picoseconds at a constant strain rate of $2 \times 10^{8} \mathrm{~s}^{-1}$. The simulations consumed a total computation time of 22.8 central processing unit years (corresponding to $3 \times 10^{18}$ flops) in the Kraken Cray XT5 system at the National Institute for Computational Sciences.

The local crystalline order method ${ }^{32}$ is used to identify defects during deformation. Five types of atoms are painted in colour: grey for perfect atoms, red for atoms in stacking faults and green for atoms in grain boundaries or dislocation cores; blue atoms indicate that they are in the vicinity of vacancies; and fully disordered atoms are yellow. Six videos are supplied in the Supplementary Information to show deformation processes in nano-twinned samples.

\section{Received 10 September 2009; accepted 16 February 2010.}

1. Hall, E. O. The deformation and ageing of mild steel: III. Discussion of results. Proc. Phys. Soc. Lond. B 64, 747-753 (1951).

2. Petch, N. J. The cleavage strength of polycrystals. J. Iron Steel Inst. 174, 25-28 (1953).

3. Hirth, J. P. \& Lothe, J. Theory of Dislocations 2nd edn, 764-790 (John Wiley and Sons, 1982).

4. Devincre, B., Hoc, T. \& Kubin, L. Dislocation mean free paths and strain hardening of crystals. Science 320, 1745-1748 (2008).

5. Meyers, M. A., Mishra, A. \& Benson, D. J. Mechanical properties of nanocrystalline materials. Prog. Mater. Sci. 51, 427-556 (2006).

6. Yip, S. Nanocrystals: the strongest size. Nature 391, 532-533 (1998).

7. Schiøtz, J., Di Tolla, F. D. \& Jacobsen, K. W. Softening of nanocrystalline metals at very small grain sizes. Nature 391, 561-563 (1998).

8. Schiøtz, J. \& Jacobsen, K. W. A maximum in the strength of nanocrystalline copper. Science 301, 1357-1359 (2003).

9. Wolf, D., Yamakov, V., Phillpot, S. R., Mukherjee, A. K. \& Gleiter, H. Deformation of nanocrystalline materials by molecular-dynamics simulation: relationship to experiments? Acta Mater. 53, 1-40 (2005).

10. Lu, L., Shen, Y., Chen, X., Qian, L. \& Lu, K. Ultrahigh strength and high electrical conductivity in copper. Science 304, 422-426 (2004).

11. Lu, L., Chen, X., Huang, X. \& Lu, K. Revealing the maximum strength in nanotwinned copper. Science 323, 607-610 (2009).
12. Cao, A. J. \& Wei, Y. G. Molecular dynamics simulation of plastic deformation of nanotwinned copper. J. Appl. Phys. 102, 083511 (2007).

13. Shabib, I. \& Miller, R. E. Deformation characteristics and stress-strain response of nanotwinned copper via molecular dynamics simulation. Acta Mater. 57, 4364-4373 (2009).

14. Chokshi, A. H., Rosen, A., Karch, J. \& Gleiter, H. On the validity of the Hall-Petch relationship in nanocrystalline materials. Scr. Metall. Mater. 23, 1679-1684 (1989).

15. Kumar, K. S., Suresh, S., Chisholm, M. F., Horton, J. A. \& Wang, P. Deformation of electrodeposited nanocrystalline nickel. Acta Mater. 51, 387-405 (2003).

16. Shan, Z. W. et al. Grain boundary-mediated plasticity in nanocrystalline nickel. Science 305, 654-657 (2004).

17. Van Swygenhoven, H., Spaczer, M., Caro, A. \& Farkas, D. Competing plastic deformation mechanisms in nanophase metals. Phys. Rev. B 60, 22-25 (1999).

18. Yamakov, V., Wolf, D., Phillpot, S. R. \& Gleiter, H. Deformation twinning in nanocrystalline Al by molecular-dynamics simulation. Acta Mater. 50, 5005-5020 (2002).

19. Uchic, M. D., Dimiduk, D. M., Florando, J. N. \& Nix, W. D. Sample dimensions influence strength and crystal plasticity. Science 305, 986-989 (2004).

20. Greer, J. R., Oliver, W. C. \& Nix, W. D. Size dependence of mechanical properties of gold at the micron scale in the absence of strain gradients. Acta Mater. 53, 1821-1830 (2005).

21. Shan, Z. W., Mishra, R. K., Syed Asif, S. A., Warren, O. L. \& Minor, A. M. Mechanical annealing and source-limited deformation in submicrometrediameter Ni crystals. Nature Mater. 7, 115-119 (2007).

22. Hemker, K. J. \& Nix, W. D. Nanoscale deformation: seeing is believing. Nature Mater. 7, 97-98 (2008).

23. Frøseth, A., Van Swygenhoven, H. \& Derlet, P. M. The influence of twins on the mechanical properties of nc-Al. Acta Mater. 52, 2259-2268 (2004).

24. Zhu, T., Li, J., Samanta, A., Kim, H. G. \& Suresh, S. Interfacial plasticity governs strain rate sensitivity and ductility in nanostructured metals. Proc. Natl Acad. Sci. USA 104, 3031-3036 (2007).

25. Christian, J. W. \& Mahajan, S. Deformation twinning. Prog. Mater. Sci. 39, 1-157 (1995).

26. Van Swygenhoven, H., Derlet, P. M. \& Froseth, A. G. Stacking fault energies and slip in nanocrystalline metals. Nature Mater. 3, 399-403 (2004).

27. Wang, Y. B., Sui, M. L. \& Ma, E. In situ observation of twin boundary migration in copper with nanoscale twins during tensile deformation. Phil. Mag. Lett. 87, 935-942 (2007).

28. Kulkarni, Y. \& Asaro, R. J. Are some nano-twinned fcc metals optimal for strength, ductility and grain stability? Acta Mater. 57, 4835-4844 (2009).

29. Mishin, Y., Mehl, M. J., Papaconstantopoulos, D. A., Voter, A. F. \& Kress, J. D. Structural stability and lattice defects in copper: ab initio, tight-binding, and embedded-atom calculations. Phys. Rev. B 63, 224106 (2001).

30. Nose, S. A. A unified formulation of the constant temperature molecular dynamics methods. J. Chem. Phys. 81, 511-519 (1984).

31. Tuckerman, M., Berne, B. J. \& Mantyna, G. J. Reversible multiple time scale molecular dynamics. J. Chem. Phys. 97, 1990-2001 (1992).

32. Van Swygenhoven, H., Farkas, D. \& Caro, A. Grain-boundary structures in polycrystalline metals at the nanoscale. Phys. Rev. B 62, 831-838 (2000).

Supplementary Information is linked to the online version of the paper at www.nature.com/nature.

Acknowledgements X.L. and H.G. acknowledge financial support by the NSF through the MRSEC Program (award number DMR-0520651) and grant CMMI-0758535 at Brown University. The simulations reported were performed on NSF TeraGrid resources provided by NICS under MSS090036 (H.G.) and DMR090083 (Y.W.), with additional support from the Computational Mechanics Research Facility at Brown University and the Alabama Supercomputer Center. Helpful discussions with J. D. Embury, W. D. Nix, H. Mughrabi, M. A. Meyers and R. Taylor are gratefully acknowledged. L.L. and K.L. acknowledge financial support by the NSFC (grant numbers 50621091, 50725103 and 50890171) and the MOST of China (grant number 2005CB623604).

Author Contributions All authors contributed equally to this work. H.G., K.L. and L.L. conceived the project. X.L. and Y.W. performed molecular dynamics simulations. All authors analysed data, developed the model, discussed the results and wrote the paper.

Author Information Reprints and permissions information is available at www.nature.com/reprints. The authors declare no competing financial interests. Correspondence and requests for materials should be addressed to Y.W. (yujie_wei@Inm.imech.ac.cn) and H.G. (huajian_gao@brown.edu). 